\title{
ON A DECOMPOSITION THEOREM FOR MEASURES IN EUCLIDEAN $n$-SPACE $\left(^{(1)}\right.$
}

\author{
BY \\ WILLIAM C. NEMITZ
}

Introduction. It is the purpose of this paper to extend a decomposition theorem of Mickle [1] (square brackets refer to the bibliography at the end of this paper) for $(n-1)$-dimensional measures in Euclidean $n$-space $R^{n}$, to $k$-dimensional measures in $R^{n}$, for $0<k<n, k$ an integer. In this paper we define a measure $\mu_{n}^{k}$ on the family $B$ of Borel sets of $R^{n}$ that satisfies the following conditions:

(a) If $B \in B$ and $\mu_{n}^{k}(B)<+\infty$, then $B=B_{1} \cup B_{2}$, where $B_{1}$ is countably $k$-rectifiable (see 4.1) and $\mu_{n}^{k}\left(B_{2}\right)=0$.

(b) If $B \in B$, then

$$
F_{n}^{k}(B) \leqq \mu_{n}^{k}(B) \leqq H_{n}^{k}(B),
$$

where $F_{n}^{k}$ is the Favard $k$-dimensional measure in $R^{n}$ (see 7.3) and $H_{n}^{k}$ is the Hausdorff $k$-measure in $R^{n}$ (see [2] 2.18). Furthermore (see 7.3),

$$
F_{n}^{k}(B)=\stackrel{k}{\mu_{n}}(B)
$$

whenever $\mu_{n}^{k}(B)<+\infty$ and

$$
F_{n}^{k}(B)=\stackrel{k}{\mu_{n}}(B)=H_{n}^{k}(B),
$$

whenever $B$ is countably $k$-rectifiable. Whether (2) holds for every $B \in B$ is an open question.

(c) $\mu_{n}^{k}$ is the smallest measure on $B$ which satisfies a weak projection inequality in the following sense: For almost every $R^{k}$ (in a sense given in 6.4), the Lebesgue $k$-dimensional measure of the projection of a Borel set $B$ into $R^{k}$ is less than or equal to $\mu_{n}^{k}(B)$.

(d) If $m$ is a positive integer such that $k<m<n$, and $B$ is a Borel set in $R^{m}$, then (see 7.4)

$$
\stackrel{k}{\mu_{m}}(B)=\stackrel{k}{\mu_{n}}(B)
$$

While our results are stated in terms of measures on Borel sets, it will be convenient to work with Borel regular Carathéodory outer measures (see

Received by the editors May 19, 1960.

(1) This work was done as a doctoral dissertation at The Ohio State University under the direction of E. J. Mickle. 
1.11). The procedures and proofs will follow closely those of Mickle [1] and Federer [2]. However, it should be noted that in the work of Mickle [1] the geometrical arguments needed do not necessitate the use of the group of orthogonal transformations of $R^{n}$ onto $R^{n}$ that is used in this paper.

I. Preliminary considerations.

1.1. Let $R^{n}$ denote Euclidean $n$-space, $B$ the Borel sets of $R^{n}, G_{n}$ the group of orthogonal $n \times n$ matrices with real entries, and $\sigma_{n}$ the Haar measure on $G_{n}$. Likewise let $G_{k}$ denote the group of orthogonal $k \times k$ matrices, and $\sigma_{k}$ the Haar measure in $G_{k}$. For $g$, an element of $G_{n}$, let $g_{i}$ be the $i$ th row vector of $g$. That is,

$$
g_{i}=\left(g_{i 1}, g_{i 2}, \cdots, g_{i n}\right),
$$

where $g_{i j}$ is the entry of $g$ in the $i$ th row and $j$ th column. Let $I^{k}$ be that $n \times n$ matrix formed from the identity of $G_{n}$ by setting the last $n-k$ diagonal elements equal to 0 . We shall use $I^{k}$ both for the above matrix and for the mapping effected by the matrix, that is, for the projection of $R^{n}$ onto the space spanned by the first $k$ basis vectors of $R^{n}$. Thus, although $I^{k}$ considered as a matrix has no inverse, we shall use $\left(I^{k}\right)^{-1}$ for the inverse of the projection mapping. For convenience, we shall set $R^{k}=I^{k}\left(R^{n}\right)$. Also let $I_{n-k}=I-I^{k}$, and $R_{n-k}=I_{n-k}\left(R^{n}\right)$. If $y$ is an element of $R^{n}$, and $g$ is an element of $G_{n}$, then the length of $I^{k} g(y)$ and $I_{n-k} g(y)$ are given by

$$
\begin{gathered}
\left|I^{k} g(y)\right|=\sum_{i=1}^{k}\left[\left(y \cdot g_{i}\right)^{2}\right]^{1 / 2}, \\
\left|I_{n \downarrow k} g(y)\right|=\sum_{i=k+1}^{n}\left[\left(y \cdot g_{i}\right)^{2}\right]^{1 / 2},
\end{gathered}
$$

where "." denotes inner product.

1.2. Let $S$ be an element of $G_{k+1}$. Then $S$ is a $k+1$ by $k+1$ orthogonal matrix. We form an $n \times n$ orthogonal matrix $S^{\prime}$ from $S$ as follows:

$$
\begin{aligned}
& S_{i}^{\prime}=\left(S_{i 1}, S_{i 2}, \cdots, S_{i, k+1}, 0, \cdots, 0\right) \quad \text { for } 1 \leqq i \leqq k+1 \text {, } \\
& S_{i}^{\prime}=\left(\delta_{i 1}, \delta_{i 2}, \cdots, \delta_{i n}\right) \quad \text { for } k+2 \leqq i \leqq n \text {. } \\
& \delta_{i j}= \begin{cases}1 & \text { for } i=j, \\
0 & \text { for } i \neq j .\end{cases}
\end{aligned}
$$

$S^{\prime}$ may be considered as a continuous function of $S$.

1.3. Lemma. For $S$ in $G_{k+1}$ and $y$ in $R^{n}$,

$$
S^{\prime} I^{k+1}(y)=I^{k+1} S^{\prime}(y) .
$$

Proof. Let 


$$
\begin{aligned}
u & =\left(u_{1}, \cdots, u_{n}\right)=S^{\prime} I^{k+1}(y), \\
v & =\left(v_{1}, \cdots, v_{n}\right)=I^{k+1} S^{\prime}(y), \\
I^{k+1}(y) & =\left(y_{1}, \cdots, y_{k+1}, 0, \cdots, 0\right) . \\
u_{i} & =\sum_{p=1}^{n} S_{i p}^{\prime}\left(I^{k+1}(y)\right)_{p}=\sum_{p=1}^{k+1} S_{i p}^{\prime} y_{p}, \\
v_{i} & =\left\{\begin{array}{cc}
\sum_{p=1}^{n} S_{i p}^{\prime} y_{p} & \text { if } 1 \leqq i \leqq k+1, \\
0 & \text { if } k+2 \leqq i \leqq n .
\end{array}\right.
\end{aligned}
$$

But if $1 \leqq i \leqq k+1$ and $k+2 \leqq p \leqq n$, then $S_{i p}^{\prime}=0$; so

$$
v_{i}=\sum_{p=1}^{k+1} S_{i p}^{\prime} y_{p}=u_{i} \quad \text { if } 1 \leqq i \leqq k+1 .
$$

Also if $k+2 \leqq i \leqq n$ and $1 \leqq p \leqq k+1$, then $S_{i p}^{\prime}=0$; so

$$
u_{i}=\sum_{p=1}^{k+1} S_{i p}^{\prime} y_{p}=0=v_{i} \quad \text { if } k+2 \leqq i \leqq n .
$$

Hence $u=v$.

1.4. Lemma. For $S$ in $G_{k+1}$ and $y$ in $R^{n}$

$$
\left|I^{k+1} S^{\prime}(y)\right|=\left|I^{k+1}(y)\right| \text {. }
$$

Proof. $S^{\prime}$ is in $G_{n}$ and hence $S^{\prime}$ preserves length. Therefore by 1.3,

$$
\left|I^{k+1} S^{\prime}(y)\right|=\left|S^{\prime} I^{k+1}(y)\right|=\left|I^{k+1}(y)\right| .
$$

1.5. Lemma. For $S$ in $G_{k+1}$ and $\alpha>0$ let

$$
\begin{aligned}
& \mathcal{U}(S, \alpha)=\left\{x\left|x \in R^{n},\right| I^{k} S^{\prime}(x) \mid<\alpha\left[S^{\prime}(x)\right] k+1\right\}, \\
& \mathcal{V}(S, \alpha)=\left\{x\left|x \in R^{n},\right| I^{k+1}(x) \mid<\left(\alpha^{2}+1\right)^{1 / 2}\left[S^{\prime}(x)\right] k+1\right\} .
\end{aligned}
$$

Then $\mathcal{u}(S, \alpha)=\mathcal{V}(S, \alpha)$.

Proof. First note that for $x$ in either set

$$
\left[S^{\prime}(x)\right]_{k+1}=\left(S_{k+1}^{\prime} \cdot x\right)>0 .
$$

Now

$$
x \in \mathfrak{u}(S, \alpha)
$$

if and only if

$$
\left|I^{k} S(x)\right|^{2}=\sum_{i=1}^{k}\left(S_{i}^{\prime} \cdot x\right)^{2}<\alpha^{2}\left[\left(S^{\prime}(x)\right)_{k+1}\right]^{2}
$$

if and only if 


$$
\left|I^{k+1} S^{\prime}(x)\right|^{2}=\sum_{i=1}^{k+1}\left(S_{i}^{\prime} \cdot x\right)^{2}<\left(\alpha^{2}+1\right)\left[\left(S^{\prime}(x)\right)_{k+1}\right]^{2} .
$$

By 1.4 then

$$
x \in \mathcal{u}(S, \alpha)
$$

if and only if

$$
\left|I^{k+1}(x)\right|^{2}<\left(\alpha^{2}+1\right)\left[\left(S^{\prime}(x)\right)_{k+1}\right]^{2}
$$

if and only if

$$
x \in \mathcal{V}(S, \alpha) .
$$

1.6. For $x$ a real number, $x>0$, let

$$
y=\beta(x)=\left\{2\left[1-\left(x^{2}+1\right)^{-1 / 2}\right]\right\}^{1 / 2} .
$$

Then $\lim _{x \rightarrow 0} \beta(x)=0$ and

$$
x=\beta^{-1}(y)=\frac{y\left(4-y^{2}\right)^{1 / 2}}{2-y^{2}}, \quad \lim _{\nu \rightarrow 0} \beta^{-1}(y)=0,
$$

and

$$
\lim _{x \rightarrow 0} \frac{\beta(x)}{x}=1
$$

1.7. Let $C^{k+1}=\left\{y\left|y \in R^{k+1},\right| y \mid=1\right\}$. For $y$ in $C^{k+1}, 0<\eta<\infty$, let

$$
\begin{aligned}
C^{0}(y, \eta) & =\left\{Z\left|Z \in C^{k+1},\right| Z-y \mid<\eta\right\}, \\
C(y, \eta) & =\left\{Z\left|Z \in C^{k+1},\right| Z-y \mid \leqq \eta\right\}, \\
D & =\left\{x\left|x \in R^{n},\right| I^{k+1}(x) \mid>0\right\} .
\end{aligned}
$$

$D$ is open in $R^{n}$.

Let $f$ be the mapping with domain $D$ and range $C^{k+1}$ given by

$$
f(x)=\frac{I^{k+1}(x)}{\left|I^{k+1}(x)\right|}
$$

$f$ is continuous onto $C^{k+1}$.

1.8. Lemma. For $S$ in $G_{k+1}, \alpha>0$,

$$
f^{-1} C^{0}\left[S_{k+1}, \beta(\alpha)\right]=V(S, \alpha) .
$$

Proof.

$$
f^{-1} C^{0}\left[S_{k+1}, \beta(\alpha)\right] \subset D .
$$

Also, for $x$ in $U(S, \alpha),\left[S^{\prime}(x)\right]_{k+1}>0$, which implies that $\left|I^{k+1} S^{\prime}(x)\right|>0$, which 
by 1.4 implies that $\left|I^{k+1}(x)\right|>0$. Hence for any $x$ under consideration, $\left|I^{k+1}(x)\right|>0$. Now by 1.3

$$
\begin{aligned}
{\left[S^{\prime}(x)\right]_{k+1} } & =\left[I^{k+1} S^{\prime}(x)\right]_{k+1} \\
& =\left[S^{\prime} I^{k+1}(x)\right]_{k+1}=S_{k+1}^{\prime} \cdot\left[I^{k+1}(x)\right] \\
& =S_{k+1} \cdot\left[I^{k+1}(x)\right]=\left(S_{k+1} \cdot f(x)\right)\left(\left|I^{k+1}(x)\right|\right) .
\end{aligned}
$$

Also

$$
\left|S_{k+1}\right|=|f(x)|=1
$$

So

$$
\left|f(x)-S_{k+1}\right|^{2}=2\left[1-S_{k+1} \cdot f(x)\right] \text {. }
$$

Thus $x$ is an element of $v(S, \alpha)$ if and only if

$$
\left|I^{k+1}(x)\right|<\left(\alpha^{2}+1\right)^{1 / 2}\left[S^{\prime}(x)\right]_{k+1} \text {, }
$$

which is true if and only if

$$
1<\left(\alpha^{2}+1\right)^{1 / 2}\left(S_{k+1} \cdot f(x)\right),
$$

which is true if and only if

$$
\left(S_{k+1} \cdot f(x)\right)>\left(\alpha^{2}+1\right)^{-1 / 2},
$$

which is true if and only if

$$
2-\left(S_{k+1} \cdot f(x)\right)<1-\left(\alpha^{2}+1\right)^{-1 / 2}=\frac{[\beta(\alpha)]^{2}}{2},
$$

which is true if and only if

$$
\left|f(x)-S_{k+1}\right|^{2}<[\beta(\alpha)]^{2} .
$$

1.9. Lemma. If $f(x)=S_{k+1}$, then $I^{k} S^{\prime}(x)=0$.

Proof. If $f(x)=S_{k+1}$ then for all $\alpha>0, f(x)$ is an element of $C^{0}\left(S_{k+1}, \beta(\alpha)\right)$, which by 1.5 and 1.8 means that $x$ is an element of $U(S, \alpha)$. Thus for all $\alpha>0$

$$
\left|I^{k} S^{\prime}(x)\right|<\alpha\left[S^{\prime}(x)\right]_{k+1}
$$

Hence

$$
\left|I^{k} S^{\prime}(x)\right|=0 \text {. }
$$

1.10. By a Carathéodory Outer Measure (abbreviated C.O.M.) on a metric space $X$, with distance $\rho$, we shall mean a non-negative set function $\Lambda$ defined for all subsets of $X$, such that

(1) $\Lambda(\varnothing)=0$ ( $\varnothing$ is the empty set),

(2) $E_{1} \subset E_{2} \subset X$ implies $\Lambda\left(E_{1}\right) \leqq \Lambda\left(E_{2}\right)$, 
(3) $E=U_{n} E_{n}$ implies $\Lambda(E) \leqq \sum_{n} \Lambda\left(E_{n}\right)$,

(4) $\rho\left(E_{1}, E_{2}\right)>0$ implies $\Lambda\left(E_{1}\right)+\Lambda\left(E_{2}\right)=\Lambda\left(E_{1} \cup E_{2}\right)$.

An outer measure is a non-negative set function defined on the subsets of $X$ which satisfies only the first three above conditions. By a $\Lambda$ measurable subset of $X$ we shall mean a set $E$ such that for all subsets $Q$ of $X$,

$$
\Lambda(Q)=\Lambda(Q \cap E)+\Lambda(Q \cap C E),
$$

where $C(E)$ denotes the complement of $E$. By a Borel regular C.O.M. we shall mean a C.O.M. such that for any subset $E$ of $X$ there is a Borel set $B$ containing $E$ for which $\Lambda(E)=\Lambda(B)$.

Let $A$ be a closed set in $R^{n}, j$ a positive integer, $\Lambda$ an outer measure in $R^{n}$. For $Y$ a subset of $C^{k+1}$, let

$$
\Psi(Y)=\underset{0<r \leqq 1 / s}{\operatorname{l.u.b.}} \frac{\Lambda\left[A \cap K(\bar{O}, r) \cap f^{-1}(Y)\right]}{\boldsymbol{r}^{k}},
$$

where $\bar{O}$ is the origin of $R^{n}$, and $K(x, r)$ is the open sphere of center $x$ and radius $r$ in $R^{n}$. Then $\psi(Y)$ is an outer measure in $C^{k+1}$.

1.11. Lemma. For $A$ closed $C R^{n}, j$ is a positive integer, $D$ as in 1.7, let

$$
Z=f(A \cap D \cap K(\bar{O}, 1 / j)) .
$$

Then $Z$ is an analytic subset of $C^{k+1}$, and

$$
\Psi\left(C^{k+1}-Z\right)=0 .
$$

Proof. Since $A \cap D \cap K(\bar{O}, 1 / j)$ is a Borel set of $R^{n}$, and $f$ is continuous, $Z$ is analytic. Also,

$$
C^{k+1}-Z=C^{k+1} \cap C f[A \cap D \cap K(\bar{O}, 1 / j)] .
$$

Hence $\Psi\left(C^{k+1}-Z\right)=$

$$
\underset{0<r \leqq 1 / j}{\operatorname{lub.b}} \frac{\Lambda\left[A \cap K(\bar{O}, r) \cap f^{-1}\left\{C^{k+1} \cap C f[A \cap D \cap K(\bar{O}, 1 / j)]\right\}\right]}{r^{k}},
$$

which is less than or equal to

$$
\underset{0<r \leqq 1 / j}{\text { l.u.b. }} \frac{\Lambda[A \cap K(\bar{O}, r) \cap D \cap C[A \cap D \cap K(\bar{O}, 1 / j)]]}{r^{k}}=0 .
$$

1.12. Lemma. Let

$$
p(y, \eta)=\frac{\Psi C(y, \eta)}{H^{k}[C(y, \eta)]}
$$

where $H^{k}$ is Hausdorff $k$ measure. Let 


$$
\begin{aligned}
& H=\left\{y \mid y \in C^{k+1}, \limsup _{\eta \rightarrow 0} p(y, \eta)=+\infty\right\}, \\
& K=\left\{y \mid y=C^{k+1}, \underset{\eta \rightarrow 0}{\lim \sup } p(y, \eta)>0\right\} .
\end{aligned}
$$

Then $H^{k}(K-(H \cup Z))=0$.

Proof. By $1.11 \Psi\left(C^{k+1}-Z\right)=0$. So $\Psi[C(y, \eta)-Z]=0$. Hence

$$
\Psi[C(y, \eta) \cap Z] \leqq \Psi[C(y, \eta)] \leqq \Psi[C(y, \eta) \cap Z]+\Psi[C(y, \eta)-Z] .
$$

Therefore

$$
p(y, \eta)=\frac{\Psi[C(y, \eta) \cap Z]}{H^{k}[C(y, \eta)]}
$$

Now, by $[3 ; 4]$,

$$
\limsup _{\eta \rightarrow 0} \frac{\Psi[C(y, \eta) \cap Z]}{H^{k}[C(y, \eta)]}=0 \text { or }+\infty,
$$

$H^{k}$ almost everywhere on $C^{k+1}-Z$. But on $K-H$

$$
0<\limsup _{\eta \rightarrow 0} p(y, \eta)<+\infty \text {. }
$$

So $H^{k}[K-(H \cup Z)]=0$.

1.13. The following are immediate consequences of 1.6 .

$$
\begin{aligned}
\lim _{\eta \rightarrow 0} \frac{H^{k}\left[C\left(y, \frac{\beta(\eta)}{2}\right)\right]}{\alpha(k) \eta^{k}} & =2^{-k}>0, \\
\lim _{\eta \rightarrow 0} \frac{H^{k}[C(y, \beta(\eta))]}{\alpha(k) \eta^{k}} & =1,
\end{aligned}
$$

where $\alpha(k)$ is the Lebesgue $k$ measure of the set

$$
\left\{x\left|x \in R^{k},\right| x \mid \leqq 1\right\} .
$$

1.14. Lemma. Let

$H^{*}(\Lambda, A)$

$$
=\left\{S \mid S \in G_{k+1}, \limsup _{\eta \rightarrow 0} \underset{0<r \leqq 1 / j}{\text { l.u.b. }} \frac{\Lambda[A \cap K(\bar{O}, r) \cap \mathcal{u}(S, \eta)]}{\alpha(k) r^{k} \eta k}=+\infty\right\} .
$$

Then $S_{k+1}$ in $H$ implies $S$ is in $H^{*}(\Lambda, A)$.

Proof. $S_{k+1}$ in $H$ implies 


$$
\underset{\eta \rightarrow 0}{\limsup } \underset{0<r \leqq 1 / s .}{\operatorname{liu.b.}} \frac{\Lambda\left[A \cap K(\bar{O}, r) \cap f^{-1} C\left(S_{k+1}, \eta\right)\right]}{r^{k} H^{k}\left[C\left(S_{k+1}, \eta\right)\right]}=+\infty .
$$

Hence by 1.6

$$
\underset{\eta \rightarrow 0}{\limsup } \underset{0<r \leq 1 / d}{\text { l.u.b. }} \frac{\Lambda\left[A \cap K(\bar{O}, r) \cap f^{-1} C\left(S_{k+1}, \beta(\eta)\right)\right]}{r^{k} H^{k}\left[C\left(S_{k+1}, \beta(\eta)\right)\right]}=+\infty .
$$

Hence since

$f^{-1}\left[C\left(S_{k+1}, \beta(\eta)\right)\right] \subset f^{-1}\left[C^{0}\left(S_{k+1}, 2 \beta(\eta)\right)\right]$,

$$
\underset{\eta \rightarrow 0}{\limsup } \underset{0<r \leqq 1 / \rho}{\text { l.u.b. }} \frac{\Lambda\left[A \cap K(\bar{O}, r) \cap f^{-1}\left[C^{0}\left(S_{k+1}, 2 \beta(\eta)\right)\right]\right]}{r^{k} H^{k}\left[C\left(S_{k+1}, \beta(\eta)\right)\right]}=+\infty,
$$

which implies that

$$
\underset{\eta \rightarrow 0}{\limsup } \underset{\substack{\text { l.u.b. } \\ 0<r \leqq 1 / s}}{\frac{\Lambda\left[A \cap K(\bar{O}, r) \cap f^{-1}\left[C^{0}\left(S_{k+1}, \beta(\eta)\right)\right]\right]}{r^{k} H^{k}\left[C\left(S_{k+1}, \frac{\beta(\eta)}{2}\right)\right]}}=+\infty .
$$

Hence by 1.14 ,

$$
+\infty=\limsup _{\eta \rightarrow 0} \underset{0<r \leqq 1 / \jmath}{\text { l.u.b. }} \frac{\Lambda\left[A \cap K(\bar{O}, r) \cap f^{-1}\left[C^{0}\left(S_{k+1}, \beta(\eta)\right)\right]\right]}{\alpha(k) r^{k} \eta^{k}},
$$

which by 1.5 and 1.8 equals

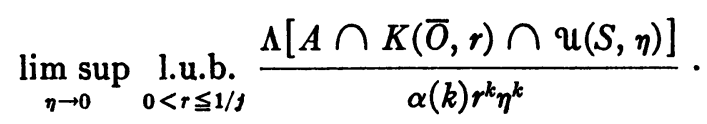

Hence $S$ is in $H^{*}(\Lambda, A)$.

1.15. Lemma. Let

$$
K^{*}(\Lambda, A)=\left\{S \mid S \in G_{k+1}, \underset{\eta \rightarrow 0}{\limsup } \underset{0<r \leqq 1 / \rho}{\text { l.u.b. }} \frac{\Lambda[A \cap K(\bar{O}, r) \cap \mathcal{u}(S, \eta)]}{\alpha(k) r^{k} \eta^{k}}>0\right\} .
$$

Then $S$ in $K^{*}(\Lambda, A)$ implies $S_{k+1}$ is in $K$.

Proof. $S$ in $K^{*}(\Lambda, A)$ implies

$$
0<\limsup \sup _{\eta \rightarrow 0} \operatorname{liu.b.}_{0<r \leqq 1 / s} \frac{\Lambda[A \cap K(\bar{O}, r) \cap u(S, \eta)]}{\alpha(k) r^{k} \eta^{k}},
$$

which by 1.5 and 1.8 is less than or equal to

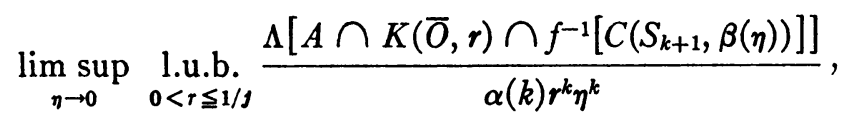


which by 1.13 is less than or equal to

$$
\begin{aligned}
& \underset{\eta \rightarrow 0}{\lim \sup } \underset{\substack{0<r \leqq 1 / \rho}}{1 . u . b .}\left\{\frac{\Lambda\left[A \cap K(\bar{O}, r) \cap f^{-1}\left[C\left(S_{k+1}, \beta(\eta)\right)\right]\right]}{\alpha(k) r^{k} \eta^{k}}\right\} \\
& \cdot\left\{\frac{\alpha(k) \eta^{k}}{B^{k}\left[C\left(S_{k+1}, \beta(\eta)\right)\right]}\right\},
\end{aligned}
$$

which by 1.6 equals $\lim \sup _{\eta \rightarrow 0} p\left(S_{k+1}, \eta\right)$. Hence $S_{k+1}$ is in $K$.

1.16. Lemma. Let

$$
E(S)=\left\{x \mid x \in R^{n}, I^{k} S^{\prime}(x)=\bar{O}\right\}
$$

Let

$$
L^{*}(A)=\left\{S \mid S \in G_{k+1},(A-\bar{O}) \cap K(\bar{O}, 1 / j) \cap E(S) \neq \varnothing\right\} .
$$

Then $S_{k+1}$ in $Z$ (see 1.11) implies that $S$ is in $L^{*}(A)$.

Proof. $S_{k+1}$ in $Z$ implies that there is an $x$ in $A \cap D \cap K(\bar{O}, 1 / j)$, such that $f(x)=S_{k+1}$. Hence by $1.9, I^{k} S^{\prime}(x)=\bar{O}$, which implies that $x$ is in $E(S)$. Now if $x$ is in $A \cap D$, then $x$ is not the origin. So $x$ is in

$$
(A-\bar{O}) \cap K(\bar{O}, 1 / j) \cap E(S)
$$

which is therefore not empty. Hence $S$ is in $L^{*}(A)$.

1.17. ThEOREM.

$$
\sigma_{k+1}\left[K^{*}(\Lambda, A)-\left(H^{*}(\Lambda, A) \cup L^{*}(A)\right)\right]=0 .
$$

Proof. By 1.15, if $S$ is in $K^{*}(\Lambda, A)$, then $S_{k+1}$ is in $K$. By 1.14, if $S$ is not in $H^{*}(\Lambda, A)$, then $S_{k+1}$ is not in $H$. By 1.16 , if $S$ is not in $L^{*}(A)$, then $S_{k+1}$ is not in $Z$. Hence

$$
K^{*}(\Lambda, A)-\left(H^{*}(\Lambda, A) \cup L^{*}(A)\right)
$$

is contained in the set of $S$ in $G_{k+1}$ such that $S_{k+1}$ is in $K-(H \cup Z)$. Hence the theorem follows from the fact that $H^{k}[K-(H \cup Z)]=0$ (see 1.12).

II. Densities.

2.1. For a fixed $\eta>0, a$ an element of $R^{n}, A$ a subset of $R^{n}, j$ a fixed positive integer, $g$ an element of $G_{n}$, and $\Lambda$ a C.O.M., let

$$
P(g, \eta, a)=\left\{x\left|x \in R^{n},\right| I^{k} g(x-a)|<\eta| I_{n-k} g(x-a) \mid\right\} .
$$

Let

$$
\begin{aligned}
& M(\Lambda, A, g, \eta, r, a)=\Lambda[A \cap K(a, r) \cap P(g, \eta, a)] / \alpha(k) \eta^{k} r^{k} ; \\
& M_{j}(\Lambda, A, g, \eta, a)=\underset{0<r \leqq 1 / j}{ } \begin{array}{l}
\text { l.u.b. } M(\Lambda, A, g, \eta, r, a) ; \\
U(g, a)=\left\{x \mid x \in R^{n}, I^{k} g(x-a)=\bar{O}\right\} ;
\end{array}
\end{aligned}
$$




$$
\begin{aligned}
W_{j}(\Lambda, A) & =\left\{(x, g) \mid(x, g) \in A \times G_{n}, \limsup _{\eta \rightarrow 0} M_{j}(\Lambda, A, g, \eta, x)=+\infty\right\} ; \\
X_{j}(\Lambda, A) & =\left\{(x, g) \mid(x, g) \in A \times G_{n}, \limsup _{\eta \rightarrow 0} M_{j}(\Lambda, A, g, \eta, x)=0\right\} ; \\
V_{j}(A) & =\left\{(x, g) \mid(x, g) \in A \times G_{n},(A-x) \cap K(x, 1 / j)\right.
\end{aligned}
$$$$
\cap U(g, x) \neq \varnothing\} \text {. }
$$

2.2. Lemma. If $A$ is closed in $R^{n}$, then $V_{j}(A)$ is a Borel set in the cartesian product space $A \times G_{n}$.

Proof. For $p$ and $q$ integers, $p$ less than $j$, and $C(x, r)$ the closed sphere for radius $r$ and center $x$ in $R^{n}$, the set $S_{j, p \cdot q}$ of elements of $A \times G_{n}$ for which

$$
A \cap[C(x, 1 / j-1 / p)-K(x,(p-j) /(j p+q)] \cap U(g, x) \neq \varnothing
$$

is a closed set in $A \times G_{n}$, and

$$
V,=\bigcup_{p=j+1}^{\infty} \bigcup_{q=1}^{\infty} S_{j, p \cdot q}
$$

2.3. Lemma. For $A$ a Borel set of $R^{n}, \Lambda$ a C.O.M., $W_{j}(\Lambda, A)$ and $X_{j}(\Lambda, A)$ are Borel sets in $A \times G_{n}$.

Proof. It suffices to show that

$$
\Lambda[A \cap K(x, r) \cap P(g, \eta, x)]
$$

is a lower semi-continuous function of $(g, \eta, r, x)$. For

$$
\left(g_{0}, \eta_{0}, r_{0}, x_{0}\right)
$$

let $\lambda$ be any number less than

$$
\Lambda\left[A \cap K\left(x_{0}, r_{0}\right) \cap P\left(g_{0}, \eta_{0}, x_{0}\right)\right] .
$$

There exists $F$, a closed set of $R^{n}$, such that

$$
F \subset K\left(x_{0}, r_{0}\right) \cap P\left(g_{0}, \eta_{0}, x_{0}\right),
$$

and

$$
\Lambda(A \cap F)>\lambda,
$$

and for $(g, \eta, r, x)$ sufficiently close to $\left(g_{0}, \eta_{0}, r_{0}, x_{0}\right)$,

$$
F \subset K(x, r) \cap P(g, \eta, x),
$$

and

$$
\Lambda[A \cap K(x, r) \cap P(g, \eta, x)] \geqq \Lambda(A \cap F)>\lambda .
$$


2.4. For $A$ a closed subset of $R^{n}$, and $x$ in $A$, the sets

$$
\begin{aligned}
W_{j}^{*}(\Lambda, A, x) & =\left\{g \mid g \in G_{n}, \underset{\eta \rightarrow 0}{\lim \sup } M_{j}(\Lambda, A, g, \eta, x)=+\infty\right\}, \\
X_{j}^{*}(\Lambda, A, x) & =\left\{g \mid g \in G_{n}, \underset{\eta \rightarrow 0}{\lim \sup } M_{j}(\Lambda, A, g, \eta, x)=0\right\}, \\
V_{j}^{*}(A, x) & =\left\{g \mid g \in G_{n},(A-x) \cap K(x, 1 / j) \cap U(g, x) \neq \varnothing\right\},
\end{aligned}
$$

are Borel sets in $G_{n}$.

2.5. For $A$ a closed subset of $R^{n}, g$ in $G_{n}$, the sets

$$
\begin{aligned}
\bar{W}_{j}(\Lambda, A, g) & =\left\{x \mid x \in R^{n}, \underset{\eta \rightarrow 0}{\lim \sup _{i}} M_{j}(\Lambda, A, g, \eta, x)=+\infty\right\}, \\
\bar{X}_{j}(\Lambda, A, g) & =\left\{x \mid x \in R^{n}, \underset{\eta \rightarrow 0}{\lim \sup _{j}} M_{j}(\Lambda, A, g, \eta, x)=0\right\}, \\
\bar{V}_{j}(A, g) & =\left\{x \mid x \in R^{n},(A-x) \cap K(x, 1 / j) \cap U(g, x) \neq \varnothing\right\}, \\
\bar{W}(\Lambda, A, g)= & \bigcap_{j} \bar{W}_{j}(\Lambda, A, g), \bar{X}(\Lambda, A, g)=\bigcup_{j} \bar{X}_{j}(\Lambda, A, g), \text { and } \\
\bar{V}(A, g) & \bigcap_{j} \bar{V}_{j}(A, g)
\end{aligned}
$$

are Borel sets in $R^{n}$. Also note that $\bar{V}(A, g)$ is the set of all points $x$ of $R^{n}$ such that $x$ is an accumulation point of $A \cap U(g, x)$.

2.6. For $g$ in $G_{n}, \eta>0$, and $a$ in $R^{n}$, let

$$
\begin{aligned}
Q_{i}^{+}(g, \eta)=\left\{x\left|x \in R^{n},\right| I^{k} g(x-a) \mid<(n-k)^{1 / 2} \eta[g(x-a)]_{i}\right\} & \text { for } k+1 \leqq i \leqq n .
\end{aligned}
$$

Likewise let

$$
\begin{array}{r}
\overline{Q_{i}}(g, \eta)=\left\{x\left|x \in R^{n},\right| I^{k} g(x-a) \mid<-(n-k)^{1 / 2} \eta[g(x-a)]_{i}\right\} \\
\text { for } k+1 \leqq i \leqq n .
\end{array}
$$

Now let

$$
F_{i}^{+}=\left\{g \mid g \in G_{n}, \limsup _{\eta \rightarrow 0} \operatorname{liu.b.~}_{0<r \leqq 1 / j} \frac{\Lambda\left[A \cap K(a, r) \cap Q_{i}^{+}(g, \eta)\right]}{\alpha(k) r^{k} \eta^{k}}>0\right\},
$$

and let

$$
F_{i}^{-}=\left\{g \mid g \in G_{n}, \limsup _{\eta \rightarrow 0} \operatorname{liu.b.}_{0<r \leqq 1 / j} \frac{\Lambda\left[A \cap K(a, r) \cap \overline{\left.Q_{i}^{-}(g, \eta)\right]}\right.}{\alpha(k) r^{k} \eta^{k}}>0\right\} .
$$


$F_{i}^{+}$and $F_{i}^{-}$are Borel sets in $G$. The proof of this is essentially the same as that given in 2.3.

2.7. Lemma.

$$
P(g, \eta, a) \subset \bigcup_{i=k+1}^{n}\left[Q_{i}^{+}(g, \eta) \cup Q_{i}^{-}(g, \eta)\right]
$$

Proof. If not, then there exists $x$ in $R^{n}$ such that

$$
\left|I^{k} g(x-a)\right|<\eta\left|I_{n-k} g(x-a)\right|
$$

and for all $i, k+1 \leqq i \leqq n$,

$$
\left|I^{k} g(x-a)\right| \geqq(n-k)^{1 / 2} \eta\left|[g(x-a)]_{i}\right| .
$$

Hence

$$
\begin{aligned}
(n-k)\left|I^{k} g(x-a)\right|^{2} & \geqq(n-k) \eta^{2}\left[\sum_{i=k+1}^{n}[g(x-a)]_{i}^{2}\right] \\
& =(n-k) \eta^{2}\left|I_{n-k} g(x-a)\right|^{2} .
\end{aligned}
$$

This is a contradiction.

2.8. LeMMA. Let $I(p, q)$ be the identity matrix with the pth row replaced by the qth row, and the qth row replaced by the negative of the pth row. Then, for $k+1 \leqq p, q \leqq n$,

$I(p, q) \cdot\left[F_{p}^{+}-\left(V_{j}^{*}(A, a) \cup W_{j}^{*}(\Lambda, A, a)\right)\right]=\overline{F_{q}}-\left(V_{j}^{*}(A, a) \cup W_{j}^{*}(\Lambda, A, a)\right)$, where "." denotes cosetting with respect to $I(p, q)$.

Proof. For $g$ in $G_{n}$, and $i \neq p, q$,

$$
[I(p q) g]_{i}=g_{i} ; \quad[I(p, q) g]_{p}=g_{q} ; \quad[I(p, q) g]_{q}=-g_{p} .
$$

Thus, since $k+1 \leqq p, q \leqq n$, we have

$$
\left|I^{k}(I(p, q) g)(x-a)\right|=\left|I^{k} g(x-a)\right|,
$$

and

$$
\left|I_{n-k}(I(p, q) g)(x-a)\right|=\left|I_{n-k} g(x-a)\right| .
$$

Thus $Q_{p}^{+}(g, \eta)=Q_{p}^{-}(I(p, q) g, \eta)$, and hence

$$
\overrightarrow{F_{q}}=I(p, q) \cdot F_{p}^{+} .
$$

Also $P(g, \eta, a)=P(I(p, q) g, \eta, a)$, and hence

$$
W_{j}^{*}(\Lambda, A, a)=I(p, q) \cdot W_{j}^{*}(\Lambda, A, a) .
$$


Also $U(g, a)=U(I(p, q) g, a)$, and hence

$$
V_{j}^{*}(A, a)=I(p, q) \cdot V_{j}^{*}(A, a) .
$$

Hence

$I(p . q) \cdot\left[F_{p}^{+}-\left(V_{j}^{*}(A, a) \cup W_{j}^{*}(\Lambda, A, a)\right)\right]=\overline{F_{j}^{-}}-\left(V_{j}^{*}(A, a) \cup W_{j}^{*}(\Lambda, A, a)\right.$.

2.9. Lemma. For $k+1 \leqq p, q \leqq n$,

$$
\sigma_{n}\left[F_{p}^{+}-\left(V_{j}^{*}(A, a) \cup W_{j}^{*}(\Lambda, A, a)\right)\right]=\sigma_{n}\left[F_{q}^{-}-\left(V_{j}^{*}(A, a) \cup W_{j}^{*}(\Lambda, A, a)\right)\right] .
$$

Proof. By 2.8

$F_{q}^{-}-\left(V_{j}^{*}(A, a) \cup W_{j}^{*}(\Lambda, A, a)\right)=I(p, q) \cdot\left[F_{p}^{+}-\left(V_{j}^{*}(A, a) \cup W_{j}^{*}(\Lambda, A, a)\right)\right]$, and the lemma follows since $\sigma_{n}$ is the Haar measure in $G_{n}$.

III. Further density considerations.

3.1. Let $A$ be a closed subset of $R^{n}, g$ an element of $G_{n}$, and $a$ an element of $A$. Let

$$
t(x)=g(x-a), \quad \text { for } x \text { an element of } R^{n} .
$$

Then $t$ is a distance preserving homeomorphism of $R^{n}$ onto $R^{n}$ such that $t(a)=\bar{O}, t K(a, r)=K(\bar{O}, r)$, and $t(A)$ is a closed subset of $R^{n}$.

3.2. Lemma. For $s$ in $G_{k+1}, \eta>0$,

$$
Q_{k+1}^{+}\left(S^{\prime} g, \eta\right)=t^{-1}\left[u\left(S,(n-k)^{1 / 2} \eta\right)\right] .
$$

Proof. $x \in Q_{k+1}^{+}\left(S^{\prime} g . \eta\right)$ if and only if

$$
\left|I^{k} S^{\prime} g(x-a)\right|<(n-k)^{1 / 2} \eta\left[S^{\prime} g(x-a)\right]_{k+1},
$$

which is true if and only if

$$
\left|I^{k} S^{\prime} g(x-a)\right|<(n-k)^{1 / 2} \eta\left[S^{\prime}(t(x))\right]_{k+1},
$$

which is true if and only if $t(x)$ is in $u\left(S,(n-k)^{1 / 2} \eta\right)$.

3.3. Lemma. For $g$ in $G_{n}, a$ in $R^{n}, S$ in $G_{k+1}, \eta>0$,

$$
t^{-1}(\mathcal{U}(S, \eta)) \subset P\left(S^{\prime} g, \eta, a\right) .
$$

Proof. If $x$ is in $t^{-1}(\mathcal{U}(S, \eta))$, then $t(x)$ is in $\mathcal{u}(S, \eta)$, and therefore

$$
\left|I^{k} S^{\prime} g(x-a)\right|<\eta\left[S^{\prime} g(x-a)\right]_{k+1},
$$

and hence 


$$
\left|I^{k} S^{\prime} g\left(x^{\prime}-a\right)\right|^{2}<\eta^{2}\left[S^{\prime} g(x-a)\right]_{k+1}^{2} \leqq \eta^{2}\left|I_{n-k} S^{\prime} g(x-a)\right|^{2},
$$

and therefore $x$ is in $P\left(S^{\prime} g, \eta, a\right)$.

3.4. Lemma. For $g$ in $G_{n}, a$ in $R^{n}, S$ in $G_{k+1}$,

$$
t^{-1}(E(S))=u\left(S^{\prime} g, a\right) .
$$

Proof. $x$ is in $t^{-1}(E(S))$ if and only if $t(x)$ is in $E(S)$, which is true if and only if

$$
\left|I^{k} S^{\prime} g(x-a)\right|=0,
$$

which is true if and only if $x$ is in $\mathcal{u}\left(S^{\prime} g, a\right)$.

3.5. Lemma. For $A$ a closed subset of $R^{n}, a$ an element of $A, g$ in $G_{n}, S$ in $G_{k+1} ; S$ is in $L^{*}(t(A))$ if and only if $S^{\prime} g$ is in $V_{j}^{*}(A, a)$.

Proof. By 3.4

$$
(A-a) \cap K(a, 1 / j) \cap u\left(S^{\prime} g, a\right)=t^{-1}[(t(A)-\bar{O}) \cap K(0,1 / j) \cap E(S)] .
$$

So $S$ is in $L^{*}(t(A))$ if and only if

$$
(t(A)-\bar{O}) \cap K(\bar{O}, 1 / j) \cap E(S) \neq \varnothing,
$$

which is true if and only if

$$
t\left[(A-a) \cap K(a, 1 / j) \cap u\left(S^{\prime} g, a\right)\right] \neq \varnothing,
$$

which is true if and only if

$$
(A-a) \cap K(a, 1 / j) \cap u\left(S^{\prime} g, a\right) \neq \varnothing,
$$

which is true if and only if $S^{\prime} g$ is in $V_{j}^{*}(A, a)$.

3.6. For $\Lambda$ an outer measure, and $E$ a subset of $R^{n}$, let

$$
\Lambda^{*}(E)=\Lambda\left[t^{-1}(E)\right]
$$

Then $\Lambda^{*}$ is an outer measure in $R^{n}$.

3.7. Lemma. For $\Lambda$ an outer measure, $A$ a closed subset of $R^{n}, g$ in $G_{n}, S$ in $G_{k+1}$; if $S$ is in $H^{*}\left(\Lambda^{*}, t(A)\right)$, then $S^{\prime} g$ is in $W_{j}^{*}(\Lambda, A, a)$.

Proof. If $S$ is in $H^{*}\left(\Lambda^{*}, t(A)\right)$, then

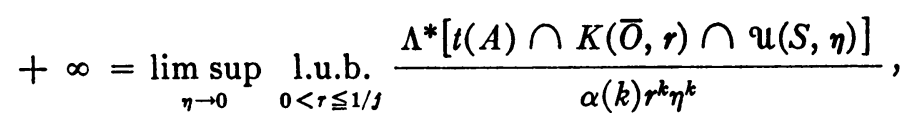

which by 3.3 is less than or equal to

$$
\underset{\eta \rightarrow 0}{\lim \sup } \underset{0<r \leqq 1 / g}{\operatorname{l.u.b.}} \frac{\Lambda\left[A \cap K(a, r) \cap P\left(S^{\prime} g, \eta, a\right)\right]}{\alpha(k) r^{k} \eta^{k}} .
$$

Hence $S^{\prime} g$ is in $W_{j}^{*}(\Lambda, A, a)$. 
3.8. Lemma. If $S^{\prime} g$ is in $F_{k+1}^{+}$, then $S$ is in $K^{*}\left(\Lambda^{*}, t(A)\right)$.

Proof. If $S^{\prime} g$ is in $F_{k+1}^{+}$, then

$$
0<\limsup \operatorname{liu.b.}_{0<r \leqq 1 / g} \frac{\Lambda\left[A \cap K(a, r) \cap Q_{k+1}^{+}\left(S^{\prime} g, \eta\right)\right]}{\alpha(k) r^{k} \eta^{k}}
$$

which by 3.2 equals

$$
\underset{\eta \rightarrow 0}{\limsup } \underset{0<r \leq 1 / j}{\text { l.u.b. }} \frac{\Lambda^{*}\left[t(A) \cap K(\bar{O}, r) \cap u\left(S,(n-k)^{1 / 2} \eta\right)\right]}{\alpha(k) r^{k} \eta^{k}},
$$

and hence $S$ is in $K^{*}\left(\Lambda^{*}, t(A)\right)$.

3.9. ThEOREM. For any $g$ in $G_{n}$, and a in $A$, a closed subset of $R^{n}$, and $\Lambda$ an outer measure,

$$
\sigma_{k+1}\left\{S \mid S^{\prime} g \in\left[F_{k+1}^{+}-\left(W_{j}^{*}(\Lambda, A, a) \cup V_{j}^{*}(A, a)\right)\right]\right\}=0 .
$$

Proof. By 3.5, 3.7, and 3.8,

$$
\begin{aligned}
\left\{S \mid S^{\prime} g \in\left[F_{k+1}^{+}-\left(W_{j}^{*}(\Lambda, A, a) \cup V_{j}^{*}(A, a)\right)\right]\right\} & \subset K^{*}\left[\Lambda^{*}, t(A)\right]-\left\{H^{*}\left[\Lambda^{*}, t(A)\right] \cup L^{*}[t(A)]\right\} .
\end{aligned}
$$

Since $\Lambda^{*}$ is an outer measure, and $t(A)$ is closed in $R^{n}$, we may apply 1.17 .

3.10. THEOREM. For $a \in A$, a closed subset of $R^{n}$, and $\Lambda$ a C.O.M.

$$
\sigma_{n}\left[F_{k+1}^{+}-\left(W_{j}^{*}(\Lambda, A, a) \cup V_{j}^{*}(A, a)\right)\right]=0 .
$$

Proof. Let $\alpha:\left(G_{k+1} \times G_{n}\right) \rightarrow G_{n}$ be a mapping defined by $\alpha(S, g)=S^{\prime} g . \alpha$ is a continuous mapping. Hence the set

$$
\begin{aligned}
\left\{(S, g) \mid(S, g) \in G_{k+1} \times G_{n}, S^{\prime} g \in[\right. & \left.\left.F_{k+1}^{+}-\left(W_{j}^{*}(\Lambda, A, a) \cup V_{j}^{*}(A, a)\right)\right]\right\} \\
& =\alpha^{-1}\left[F_{k+1}^{+}-\left(W_{j}^{*}(\Lambda, A, a) \cup V_{j}^{*}(A, a)\right)\right]
\end{aligned}
$$

is a Borel set in $G_{k+1} \times G_{n}$.

Let $c(S, g)$ equal 1 if $S^{\prime} g$ is in $F_{x+1}^{+}-\left(W_{j}^{*}(\Lambda, A, a) \cup V_{j}^{*}(A, a)\right)$, and 0 otherwise. Then, since $\sigma_{n}$ is a Haar measure in $G_{n}$, for any $S$ in $G_{k+1}$,

$$
\begin{aligned}
\sigma_{n}\left[F_{k+1}^{+}-\right. & \left.\left(W_{j}^{*}(\Lambda, A, a) \cup V_{j}^{*}(A, a)\right)\right] \\
& =\sigma_{n}\left\{g \mid S^{\prime} g \in\left[F_{k+1}^{+}-\left(W_{j}^{*}(\Lambda, A, a) \cup V_{j}^{*}(A, a)\right)\right]\right\}=\int_{a_{n}} c(S, g) d \sigma_{n} .
\end{aligned}
$$

So by 3.9 


$$
\begin{aligned}
\sigma_{n}\left[F_{k+1}^{+}-\left(W_{j}^{*}(\Lambda, A, a) \cup V_{j}^{*}(A, a)\right)\right] \\
=\int_{G_{k+1}} \int_{G_{n}} c(S, g) d \sigma_{n} d \sigma_{k+1}=\int_{G_{n}} \int_{G_{k+1}} c(S, g) d \sigma_{k+1} d \sigma_{n}=0 .
\end{aligned}
$$

3.11. Lemma. For $k+1 \leqq p, q \leqq n, a, A$, and $\Lambda$ as in 3.10 ,

$$
\sigma_{n}\left[F_{p}^{+}-\left(W_{j}^{*}(\Lambda, A, a) \cup V_{j}^{*}(A, a)\right)\right]=\sigma_{n}\left[\overline{F_{q}^{-}}-\left(W_{j}^{*}(\Lambda, A, a) \cup V_{j}^{*}(A, a)\right)\right]=0 .
$$

Proof. By 2.8 and 3.10,

$$
\begin{array}{r}
\sigma_{n}\left[F_{p}^{+}-\left(W_{j}^{*}(\Lambda, A, a) \cup V_{j}^{*}(A, a)\right)\right]=\sigma_{n}\left[\overline{F_{q}^{-}}-\left(W_{j}^{*}(\Lambda, A, a) \cup V_{j}^{*}(A, a)\right)\right] \\
=\sigma_{n}\left[F_{k+1}^{+}-\left(W_{j}^{*}(\Lambda, A, a) \cup V_{j}^{*}(A, a)\right)\right]=0 .
\end{array}
$$

3.12. Lemma. For $a, A$, and $\Lambda$ as in 3.10

$$
G_{n}-X_{j}^{*}(\Lambda, A, a) \subset \bigcup_{i=k+1}^{n}\left(F_{i}^{+} \cup \overline{F_{i}}\right) .
$$

Proof. If not, then there exists a $g$ in $G_{n}$, such that

$$
\underset{\eta \rightarrow 0}{\lim \sup } M_{j}(\Lambda, A, g, \eta, a)>0,
$$

and for all $i, k+1 \leqq i \leqq n$,

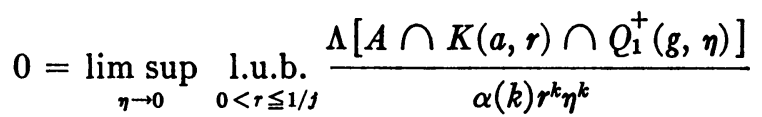

$$
\begin{aligned}
& =\limsup _{\eta \rightarrow 0} \operatorname{llu.b.}_{0<r \leqq 1 / j} \frac{\Lambda\left[A \cap K(a, r) \cap \overline{Q_{i}}(g, \eta)\right]}{\alpha(k) r^{k} \eta^{k}} .
\end{aligned}
$$

Hence by 2.7 ,

$$
\begin{aligned}
& 0<\limsup \operatorname{lic}_{\eta \rightarrow 0}^{\text {l.u.b. }} \frac{\Lambda[A \cap K(a, r) \cap P(g, \eta, a)]}{\alpha(k) r^{k} \eta^{k}} \\
& \leqq \sum_{i=k+1}^{n}\left[\lim \sup \underset{\eta \rightarrow 0}{\operatorname{liu.b}} \frac{\Lambda\left[A \cap K(a, r) \cap Q_{i}^{+}(g, \eta)\right]}{\alpha(k) r^{k} \eta^{k}}\right. \\
& \left.+\lim \sup \underset{\eta \rightarrow 0}{ } \operatorname{liu.b.}_{0<r \leqq 1 / j} \frac{\Lambda\left[A \cap K(a, r) \cap \overline{Q_{i}}(g, \eta)\right]}{\alpha(k) r^{k} \eta^{k}}\right]=0 .
\end{aligned}
$$

This is a contradiction.

3.13. Lemma. For $a, A$ and $\Lambda$ as in 3.10

$$
\sigma_{n}\left[G_{n}-\left(X_{j}^{*}(\Lambda, A, a) \cup W_{j}^{*}(\Lambda, A, a) \cup V_{j}^{*}(A, a)\right]=0 .\right.
$$


Proof. By 3.12

$$
\begin{aligned}
& G_{n}-\left(X_{j}^{*}(\Lambda, A, a) \cup W_{j}^{*}(\Lambda, A, a) \cup V_{j}^{*}(A, a)\right) \\
& \subset \bigcup_{i=k+1}^{n}\left[F_{i}^{+}-\left(W_{j}^{*}(\Lambda, A, a) \cup V_{j}^{*}(A, a)\right)\right] \cup\left[F_{i}^{-}-\left(W_{j}^{*}(\Lambda, A, a) \cup V_{j}^{*}(A, a)\right)\right],
\end{aligned}
$$

and the result follows from 3.11 .

3.14. Lemma. For $A$, a closed subset of $R^{n}, j$ an integer, $\Lambda$ a C.O.M.,

$$
\Lambda\left[A-\left(\bar{W}_{j}(\Lambda, A, g) \cup \bar{X}_{j}(\Lambda, A, g) \cup \bar{V}_{j}(A, g)\right)\right]=0
$$

for $\sigma_{n}$ almost every $g$ in $G_{n}$.

Proof. By 3.13 for any $a$ in $A$,

$$
\sigma_{n}\left[G_{n}-\left(W_{j}^{*}(\Lambda, A, a) \cup X_{j}^{*}(\Lambda, A, g) \cup V_{j}^{*}(A, a)\right)\right]=0 .
$$

Apply Fubini's theorem to the characteristic function of

$$
\left(A \times G_{n}\right)-\left(W_{j}(\Lambda, A) \cup X_{j}(\Lambda, A) \cup V_{j}(A)\right) \text {. }
$$

3.15. Theorem. For $A$ a closed subset of $R^{n}, \Lambda$ a C.O.M.

$$
\Lambda[A-(\bar{W}(\Lambda, A, g) \cup \bar{X}(\Lambda, A, g) \cup \bar{V}(A, g))]=0,
$$

for $\sigma_{n}$ almost every $g$ in $G_{n}$.

Proof. By 3.14, for every integer $j$, there is a subset $Z_{j}$ of $G_{n}$, such that $\sigma_{n}(Z)=$,0 , and for $g$ in $G_{n}-Z_{j}$

$$
\Lambda\left[A-\left(\bar{W}_{j}(\Lambda, A, g) \cup \bar{X}_{j}(\Lambda, A, g) \cup \bar{V}_{j}(A, g)\right)\right]=0 .
$$

Let $Z=\bigcup_{j=1}^{\infty} Z_{j}$. Then $\sigma_{n}(Z)=0$, and for $g$ in $G_{n}-Z$, and for all integers $j$,

$$
\Lambda\left[A-\left(\bar{W}_{j}(\Lambda, A, g) \cup \bar{X}_{j}(\Lambda, A, g) \cup \bar{V}_{j}(A, g)\right)\right]=0,
$$

and thus, by 2.5 ,

$$
\Lambda\left[A-\left(\bar{W}_{j}(\Lambda, A, g) \cup \bar{X}(\Lambda, A, g) \cup \bar{V}_{j}(A, g)\right)\right]=0 .
$$

Also, $\bar{W}_{j}(\Lambda, A, g)$ and $\bar{V}_{j}(A, g)$ are monotone decreasing sequences of sets. So

$$
\begin{aligned}
A- & (\bar{W}(\Lambda, A, g) \cup \bar{X}(\Lambda, A, g) \cup \bar{V}(A, g)) \\
= & {\left.[A-\bar{X}(\Lambda A, g)] \cap \bigcup_{j} C \bar{W}_{j}(\Lambda, A, g)\right] \cap\left[\bigcup_{j} C \bar{V}_{j}(A, g)\right] } \\
& \subset[A-\bar{X}(\Lambda, A, g)] \cap \bigcup_{j}\left[C \bar{W}_{j}(\Lambda, A, g) \cap C \bar{V}_{j}(A, g)\right] \\
& =\bigcup_{j}\left[A-\bar{X}(\Lambda, A, g) \cup \bar{W}_{j}(\Lambda, A, g) \cup \bar{V}_{j}(A, g)\right],
\end{aligned}
$$


and the result follows.

IV. $(\Lambda, k)$ Unrectifiable sets.

4.1. By a Lipschitz mapping $T$ from a metric space $X$, with metric $\rho$, to a metric space $Y$, with metric $\delta$, we shall mean a mapping such that there exists a constant $M$ such that for all $x, y$ in $X, \delta[T(x), T(y)] \leqq M \rho(x, y)$.

A subset of Euclidean $n$-space will be said to be $k$-rectifiable if it is the image under a Lipschitz mapping of a bounded subset of Euclidean $k$-space.

If $\Lambda$ is an outer measure on Eucidean $n$-space, a subset $E$ of $n$-space will be said to be $(\Lambda, k)$ unrectifiable if every $k$-rectifiable subset of $E$ is of $\Lambda$ measure 0 .

LEMMA. Let $\Lambda$ be an outer measure in $R^{n}$. Let $A$ be a subset of $R^{n}$ such that $d(A)$, the diameter of $A$ is less than $1 / j$, for a fixed integer $j$. Let $g$ be an element of $G_{n}, 0<\delta<1,0<\gamma<+\infty$, and assume that

$$
M_{j}(\Lambda, A, g, \eta, x)<\gamma \quad \text { for } x \text { in } A \text { and } 0<\eta<\delta .
$$

Let

(2) $B=\{x \mid x \in A, A \cap P(g, \eta, x) \cap K(x, r) \neq \varnothing$ for $r>0,0<\eta<1\}$.

For $x$ in $R^{n}$, let

$$
x^{\prime}=I_{n-k} g(x)
$$

and let

$$
x^{\prime \prime}=I^{k} g(x)
$$

Also let

$$
K^{\prime \prime}\left(x^{\prime \prime}, r\right)=I^{k} g(K(x, r))
$$

Then for $a$ in $B$, we have

$$
\Lambda\left[B \cap\left(I^{k} g\right)^{-1}\left(K^{\prime \prime}\left(a^{\prime \prime}, r\right)\right)\right]<2^{k+1} 10^{2 k} \gamma \alpha(k) r^{k},
$$

for $0<r<\delta / 12 j$.

Proof. Since $d(A)<1 / j$, (1) implies that

$$
M(\Lambda, A, g, \eta, r, x)<\gamma \quad \text { for } x \text { in } A, 0<\eta<\delta, r>0 .
$$

Now for $x, y$ in $R^{n}$

$$
\begin{aligned}
\left|y^{\prime \prime}-x^{\prime \prime}\right| & =\left|I^{k} g(y)-I^{k} g(x)\right|=\left|I^{k} g(y-x)\right|, \\
\left|y^{\prime}-x^{\prime}\right| & =\left|I_{n-k} g(y)-I_{n-k} g(x)\right|=\left|I_{n-k} g(y-x)\right| .
\end{aligned}
$$

With this remark, the proof proceeds exactly as in ([1], 5.2). For $a$ in $B$, $0<r<\delta / 12 j$, let $\eta=12 r j, \epsilon=\eta / 12=r j$. Set

$$
E=B \cap\left(I^{k} g\right)^{-1}\left(K^{\prime \prime}\left(a^{\prime \prime}, r\right)\right) .
$$


For $x$ in $E$, set $h(x)=1$. u.b. $\left|y^{\prime}-x^{\prime}\right|$ for $y$ in

$$
A \cap P(g, \epsilon, x) \cap\left(I^{k} g\right)^{-1}\left(K^{\prime \prime}\left(a^{\prime \prime}, r\right)\right) .
$$

Then $0<h(x) \leqq 1 / j$. For $x$ in $E$, let $\bar{x}$ in $A \cap P(g, \epsilon, x) \cap\left(I^{k} g\right)^{-1}\left(K^{\prime \prime}\left(a^{\prime \prime}, r\right)\right)$ be such that

$$
12\left|\bar{x}^{\prime}-x^{\prime}\right|>11 h(x)
$$

Since $\bar{x}$ is in $P(g, \epsilon, x)$,

$$
\left|\bar{x}^{\prime \prime}-x^{\prime \prime}\right|<\epsilon\left|\bar{x}^{\prime}-x^{\prime}\right| \text {. }
$$

For $x$ in $E$, set

$$
Q(x) \text { equal the set of } y \text { in } E \text { such that }
$$

$$
\left|y^{\prime \prime}-x^{\prime \prime}\right|<5 \epsilon h(x) \text {. }
$$

The remainder of the proof is divided into five parts.

PART 1. For $x$ in $E, Q(x) \subset P(g, \eta, x) \cup P(g, \eta, \bar{x})$.

Proof. If not, then there is an $x$ in $E$, such that

$$
\left|y^{\prime \prime}-x^{\prime \prime}\right|<5 \epsilon h(x),\left|y^{\prime \prime}-x^{\prime \prime}\right| \geqq \eta\left|y^{\prime}-x^{\prime}\right|,\left|y^{\prime \prime}-\bar{x}^{\prime \prime}\right| \geqq \eta\left|y^{\prime}-\bar{x}^{\prime}\right| \text {. }
$$

Then

$$
\begin{aligned}
0< & 11 \epsilon h(x)<12 \epsilon\left|\bar{x}^{\prime}-x^{\prime}\right|=\eta\left|\bar{x}^{\prime}-x^{\prime}\right| \leqq \eta\left|\bar{x}^{\prime}-y^{\prime}\right|+\eta\left|y^{\prime}-x^{\prime}\right| \\
\leqq & \left|y^{\prime \prime}-\bar{x}^{\prime \prime}\right|+\left|y^{\prime \prime}-x^{\prime \prime}\right| \leqq\left|x^{\prime \prime}-\bar{x}^{\prime \prime}\right|+2\left|y^{\prime \prime}-x^{\prime \prime}\right|<\epsilon\left|x^{\prime}-\bar{x}^{\prime}\right| \\
& +10 \epsilon h(x) \leqq 11 \epsilon h(x) .
\end{aligned}
$$

This is a contradiction.

PART 2. For $x$ in $E, K^{\prime \prime}\left(x^{\prime \prime}, \epsilon h(x)\right) \subset K^{\prime \prime}\left(a^{\prime \prime}, 2 r\right)$.

Proof. If $\left|y^{\prime \prime}-x^{\prime \prime}\right|<\epsilon h(x)$, then, since $\left|x^{\prime \prime}-a^{\prime \prime}\right|<r$,

$$
\left|y^{\prime \prime}-a^{\prime \prime}\right|<\left|y^{\prime \prime}-x^{\prime \prime}\right|+\left|x^{\prime \prime}-a^{\prime \prime}\right|<\epsilon h(x)+r<2 r .
$$

Part 3. If $y$ is in $Q(x)$, then $\left|y^{\prime}-x^{\prime}\right|<5 h(x)$.

Proof. Assume that there is a $y$ in $Q(x)$ such that $\left|y^{\prime}-x^{\prime}\right| \geqq 5 h(x)$. Then $\left|y^{\prime \prime}-x^{\prime \prime}\right|<5 \epsilon h(x) \leqq \epsilon\left|y^{\prime}-x^{\prime}\right|$. Thus $y$ is in $A \cap P(g, \epsilon, x) \cap\left(I^{k} g\right)^{-1}\left(K\left(a^{\prime \prime}, r\right)\right)$, and hence $1 / j \geqq h(x) \geqq 5 h(x)>0$. This is a contradiction.

PART 4. If $x$ is in $E$, then $\Lambda(Q(x)) \leqq 2(84)^{k} \gamma \alpha(k)[\epsilon h(x)]^{k}$.

Proof. By Part 3, if $y$ is in $Q(x)$, then

$|y-x| \leqq\left|y^{\prime}-x^{\prime}\right|+\left|y^{\prime \prime}-x^{\prime \prime}\right|<5 h(x)+5 \epsilon h(x)=5(\epsilon+1) h(x)<6 h(x)$.

Also

$$
\begin{aligned}
|y-\bar{x}| & \leqq|y-x|+|x-\bar{x}| \leqq 5(\epsilon+1) h(x)+\left|x^{\prime}-\bar{x}^{\prime}\right|+\left|x^{\prime \prime}-\bar{x}^{\prime \prime}\right| \\
& <5(\epsilon+1) h(x)+(\epsilon+1)\left|x^{\prime}-\bar{x}\right|<6(\epsilon+1) h(x) \\
& <(13 / 12) \cdot 6 h(x)<7 h(x) .
\end{aligned}
$$


Hence

$$
Q(x) \subset K(x, 7 h(x)) \cap K(\bar{x}, 7 h(x)) .
$$

Hence, by Part 1 ,

$Q(x) \subset[A \cap P(g, \eta, x) \cap K(x, 7 h(x))] \cup[A \cap P(g, \eta, \bar{x}) \cap K(\bar{x}, 7 h(x))]$.

Thus by (4) and since

$$
\eta=12 \epsilon, \Lambda[Q(x)] \leqq 2 \gamma \alpha(k)(7 h(x) \eta)^{k}=2(84)^{k} \gamma \alpha(k)(\epsilon h(x))^{k} .
$$

PART 5. $\Lambda(E) \leqq 2^{k+1} 10^{2 k} \alpha(k) \gamma r^{k}$.

Proof. Since, for $x$ in $E, I^{k} g(E) \subset U K^{\prime \prime}\left(x^{\prime \prime}, \epsilon h(x)\right)$, by a covering theorem of Morse, [5], there are $x_{1}, x_{2}, \cdots$ in $E$ such that $I^{k} g(E) \subset \bigcup_{i=1}^{\infty} K^{\prime \prime}\left(x_{i}^{\prime \prime}, 5 \epsilon h\left(x_{k}\right)\right)$, and for $p \neq q$

$$
K^{\prime \prime}\left(x_{p}^{\prime \prime}, \epsilon h\left(x_{p}\right)\right) \cap K^{\prime \prime}\left(x_{q}^{\prime \prime}, \epsilon h\left(x_{q}\right)\right)=\varnothing .
$$

Now for $x$ in $E$, there is an $x_{i}$ such that $\left|x^{\prime \prime}-x_{i}^{\prime \prime}\right|<5 \epsilon h(x)$, and hence $x$ is in $Q\left(x_{i}\right)$. Thus

$$
E \subset \bigcup_{i=1}^{\infty} Q\left(x_{i}\right)
$$

From Parts 2 and 4,

$$
\begin{aligned}
\Lambda(E) & \leqq \sum_{i=1}^{\infty} \Lambda\left(Q\left(x_{i}\right)\right) \leqq 2(84)^{k} \alpha(k) \gamma \sum_{i=1}^{\infty}\left(\epsilon h\left(x_{i}\right)\right)^{k} \\
& \leqq 2(84)^{k}(2)^{k} \gamma \alpha(k) r^{k} \leqq 2^{k+1} 10^{2 k} \gamma \alpha(k) r^{k}
\end{aligned}
$$

4.2. Theorem. Let $\Lambda$ be a C.O.M. in $R^{n}, g$ in $G_{n}$, and let $A$ be $a(\Lambda, k)$ unrectifiable subset of $R$. Then (see 2.5)

$$
\Lambda[\bar{X}(\Lambda, A, g)]=0 .
$$

Proof. It suffices to show that for $j$ an integer $\Lambda\left[\bar{X}_{j}(\Lambda, A, g)\right]=0$. Also, since $R^{n}$ is separable, we may assume $d\left(\bar{X}_{j}(\Lambda, A, g)\right)<1 / j$. Now $\lim \sup _{\eta \rightarrow 0} M_{j}\left(\Lambda, \bar{X}_{j}, g, \eta, x\right)=0$ for $x$ in $\bar{X}_{j}$. Assume $\Lambda\left[\bar{X}_{j}(\Lambda, A, g)\right]>0$. Hence by 2.5 , there is a set $\bar{A}_{j}$ contained in $\bar{X}_{j}$, and a sequence of positive $n$ numbers $\delta_{1}, \delta_{2}, \cdots$, such that

$$
\Lambda\left(\bar{A}_{j}\right)>0, \bar{M}_{j}\left(\Lambda, \bar{A}_{j}, g, \eta, x\right)<1 / i, \quad \text { for } 0<\eta<\delta_{i}
$$

and $x$ in $\bar{A}_{j}$. Let $B_{j}$ be the set of $x$ in $\bar{A}_{j}$, such that for $r>0,0<\eta<1$,

$$
\bar{A}_{j} \cap P(g, \eta, x) \cap K(x, r) \neq \varnothing .
$$

Since $\bar{A}_{j}$ is $(\Lambda, k)$ unrectifiable, by Federer ([2] 4.3 Theorem),

$$
\Lambda\left(\bar{A}_{j}-B_{j}\right)=0, \quad \Lambda\left[B_{j}\right]=\Lambda\left[\bar{A}_{j}\right]>0 .
$$


Thus there is a point $a$ in $B_{j}$ such that

$$
\limsup _{r \rightarrow 0} \Lambda\left[B \cap\left(I^{k} g\right)^{-1}\left(K^{\prime \prime}\left(a^{\prime \prime}, r\right)\right)\right] / \alpha(k) r^{k}>\lambda>0,
$$

for some $\lambda$. Choose $i$ such that $1 / i<\lambda / 2^{k+1} 10^{2 k}$. By 4.1 ,

$$
\Delta\left[B \cap\left(I^{k} g\right)^{-1}\left(K^{\prime \prime}\left(a^{\prime \prime}, r\right)\right)\right]<\alpha(k) r^{k} \lambda,
$$

for $0<r<\delta_{i} / 12 j$.

Since (1) contradicts (2), it follows that

$$
\Lambda\left(\bar{X}_{j}(\Lambda, A, g)\right)=0 .
$$

\section{Lebesgue measure of projected sets.}

5.1. Lemma. If $\Lambda$ is a C.O.M. in $R^{n}, g$ in $G_{n}$, and $A$ is a subset of $R^{n}$, with $\Lambda(A)<+\infty$, then,

$$
L_{k}\left[I^{k} g(\bar{W}(\Lambda, A, g))\right]=0,
$$

where $L_{k}$ denotes Lebesgue measure in $R^{k}$.

Proof. For $E$ in $R^{k}$, let

$$
\Lambda_{0}(E)=\Lambda\left[A \cap\left(I^{k} g\right)^{-1}(E)\right] .
$$

Then $\Lambda_{\theta}$ is finite-valued C.O.M. in $R^{k}$, and for $x^{\prime \prime}$ in $R^{k}$

$$
\limsup _{t \rightarrow 0} \Lambda_{\theta}\left(K^{\prime \prime}\left(x^{\prime \prime}, t\right)\right) / \alpha(k) t^{k}<+\infty,
$$

for $L_{k}$ almost every $x^{\prime \prime}$ in $R^{k}$. Now for $x$ in $\bar{W}(\Lambda, A, g)$, and $0<\eta<1,0<r$ $<+\infty$, the set $P(g, \eta, x) \cap K(x, r)$ is contained in the set of $y$ in $R^{n}$ such that $y^{\prime \prime}$ is an element of $K^{\prime \prime}\left(x^{\prime \prime}, \eta r\right)$. Thus there is a sequence

$$
\left(\eta_{i}, r_{i}\right) \rightarrow(0,0) \quad \text { for } i \rightarrow+\infty,
$$

such that

$$
+\infty=\lim _{i \rightarrow \infty} M\left(\Lambda, A, g, \eta_{i}, r_{i}, x\right)=\lim _{i \rightarrow \infty} \Lambda_{\theta}\left(K^{\prime \prime}\left(x^{\prime \prime}, \eta_{i} r_{i}\right)\right) / \alpha(k) \eta_{i}^{k} \boldsymbol{\eta}_{i}^{k},
$$

and therefore the result follows from (1).

5.2. Lemma. Let $\Lambda$ be a C.O.M. in $R^{n}, g$ in $G_{n}$, and $A$ a Borel subset of $R^{n}$ such that

$$
\Lambda(A)<+\infty
$$

and

$$
\Lambda(A) \geqq \int_{R_{k}} N\left(x^{\prime \prime}, I^{k} g, A\right) d L_{k},
$$


where $N\left(x^{\prime \prime}, I^{k} g, A\right)$ is the number of points (possibly $\left.\infty\right)$ in $A \cap\left(I^{k} g\right)^{-1}\left(x^{\prime \prime}\right)$. Then

$$
L_{k}\left(I^{k} g(\bar{V}(A, g))\right)=0 .
$$

Proof. $N\left(x^{\prime \prime}, I^{k} g, A\right)$ is finite for $L_{k}$ almost every $x^{\prime \prime}$ in $R^{k}$. But $x$ in $\bar{V}(A, g)$ implies $N\left(x^{\prime \prime}, I^{k} g, A\right)$ is infinite. Hence the result follows.

VI. The $\mu_{n}^{k}$ measure.

6.1. Let $Z$ denote the family of subsets $Z$ of $G_{n}$, such that $\sigma_{n}(Z)=0$. For $B$ a Borel subset of $R^{n}$, and $Z$ in $\mathrm{Z}$, let

$$
\lambda_{z}^{*}(B)=\text { l.u.b. } L_{k}\left[I^{k} g(B)\right], \quad \text { for } g \text { in } G_{n}-Z \text {. }
$$

Let

$$
\lambda(B)=\text { g.l.b. } \lambda_{Z}^{*}(B), \quad \text { for } Z \text { in } \mathbf{Z} \text {. }
$$

For $E$ a subset of $R^{n}$, and $\epsilon>0$, let $\mu_{\epsilon}(E)=$ g.l.b. $\sum \lambda\left(B_{i}\right)$, the sum being taken over a countable covering of $E$ by Borel sets $B_{i}$ such that $d\left(B_{i}\right)<\epsilon$, and the greatest lower bound being taken over the family of all such coverings.

For $E$ a subset of $R^{n}$, let

$$
\stackrel{k}{\mu_{n}}(E)=\lim _{\epsilon \rightarrow 0} \mu_{\epsilon}(E)
$$

$\mu_{n}^{k}$ is a Borel regular C.O.M. in $R^{n}$.

6.2. Lemma. Let $B_{i}$ be Borel sets for $i=0,1,2, \cdots$, and assume $B_{0} \subset \cup_{i} B_{i}$, and $Z$ is in $\mathrm{Z}$. Then

$$
\lambda_{Z}^{*}\left(B_{0}\right) \leqq \sum_{i} \lambda_{Z}^{*}\left(B_{\imath}\right)
$$

Proof.

$$
\text { l.u.b. } \begin{aligned}
L_{k}\left[I^{k} g\left(B_{0}\right)\right] & \leqq \text { l.u.b. } L_{k}\left[\bigcup_{i}\left[I^{k} g\left(B_{i}\right)\right]\right] \leqq \text { l.u.b. } \sum_{i} L_{k}\left[I^{k} g\left(B_{i}\right)\right] \\
& \leqq \sum_{i} \text { l.u.b. } L_{k}\left[I^{k} g\left(B_{i}\right)\right]
\end{aligned}
$$

all least upper bounds being taken over $g$ in $G_{n}-Z$.

6.3. Lemma. For $B$ a Borel set in $R^{n}$, there is a set $Z$ in $\mathrm{Z}$ such that $\lambda(B)$ $=\lambda_{2}^{*}(B)$.

Proof. For any integer $i$, there is a set $Z_{i}$ in $\mathrm{Z}$ such that $\lambda_{Z_{i}}^{*}(B)<\lambda(B)$ $+1 / i$. Let $Z=U_{i} Z_{i}$. $Z$ is in $Z$, and $\lambda(B) \leqq \lambda_{Z}^{*}(B)<\lambda_{Z_{i}}^{*}(B)<\lambda(B)+1 / i$. Hence for all $i, \lambda(B) \leqq \lambda_{Z}^{*}(B)<\lambda(B)+1 / i$. Therefore $\lambda(B)=\lambda_{Z}^{*}(B)$. 
6.4. Lemma. For $E \subset R^{n}$, there is a set $Z$ in $Z$ such that for $g$ in $G_{n}-Z$, $\mu_{n}^{k}(E) \geqq L_{k} I^{k} g(E)$.

Proof. Since $\mu_{n}^{k}$ is Borel regular, it suffices to work with Borel sets. Let $B$ be a Borel set, $\epsilon>0$, and $i$ an integer. There is a countable covering of $B$ by Borel sets $B_{j}, d\left(B_{j}\right)>\epsilon$, such that $\mu_{n}^{k}(B)+1 / i>\sum_{i=1}^{\infty} \lambda\left(B_{j}\right)$. By 6.3 for each $j$ there is a $Z_{j}$ in $\mathbf{Z}$ such that

$$
\lambda\left(B_{j}\right)=\lambda^{*} Z_{j}\left(B_{j}\right) .
$$

Set $Z^{i}=\bigcup_{j=1}^{\infty} Z_{j}$. Then $Z^{i}$ is in $Z$ and, (by 6.2)

$$
\mu_{n}^{k}(B)+1 / i>\sum_{i=1}^{\infty} \lambda_{z}^{*} i\left(B_{j}\right) \geqq \lambda_{z}^{*} i(B) .
$$

Now set $Z=\bigcup_{i=1}^{\infty} Z^{i}$. Then $Z$ is in $Z$, and

$$
\stackrel{k}{\mu_{n}}(B)+1 / i>\lambda_{z}^{*}(B) \geqq L_{k} I^{k} g(B),
$$

for $g$ in $G_{n}-Z$, and for all $i$. Hence $\mu_{n}^{k}(B) \geqq L_{k} I^{k} g(B)$ for $g$ in $G_{n}-Z$.

6.5. Lemma. Let $\Lambda$ be a Borel regular C.O.M. in $R^{n}$. Assume that for any subset $E$ of $R^{n}$, there is a set $Z$ in $Z$ such that for $g$ in $G_{n}-Z, \Lambda(E) \geqq L_{k} I^{k} g(E)$. Then $\Lambda(E) \geqq \mu_{n}^{k}(E)$.

Proof. Again it suffices to work only with Borel sets $B$. For $\epsilon>0, B$ may be written as a countable union of Borel sets $B_{i}$, where $d\left(B_{i}\right)<\epsilon$, and for $i \neq j, B_{i} \cap B_{j}=\varnothing$. Further for all integers $i$, there is a set $Z_{i}$ in $Z$ such that for $g$ in $G_{n}-Z_{i} . \Lambda\left(B_{i}\right) \geqq L_{k} I^{k} g\left(B_{i}\right)$. Set $Z=\bigcup_{i=1}^{\infty} Z_{i}$. Then $Z$ is in $Z$, and $\Lambda\left(B_{i}\right)$ $\geqq$ l.u.b. $\left[L_{k} I^{k} g\left(B_{i}\right)\right] \geqq \lambda\left(B_{i}\right)$ for $g$ in $G_{n}-Z$. Hence $\Lambda(B) \geqq \sum_{i=1}^{\infty} \lambda\left(B_{i}\right) \geqq \mu_{e}(B)$.

The result follows since $\epsilon$ was arbitrary.

6.6. Lemma. Let $\Lambda$ be a Borel regular C.O.M. in $R^{n}$. Let $\Gamma$ be a Borel regular C.O.M. in $R^{k}$ such that for all $E \subset R^{n}$, such that $\Lambda(E)<+\infty$, there is a set $Z_{B}$ in $\mathrm{Z}$ such that for $\mathrm{g}$ in $G-Z_{E}, \Lambda(E) \geqq \Gamma I^{k} g(E)$. Then for all $E \subset R^{n}$, such that $\Lambda(E)<+\infty$, there is a set $Z$ in $Z$ such that for all subsets $E^{*} C E$, and $g$ in $G_{n}-Z$,

$$
\Lambda\left(E^{*}\right) \geqq \Gamma\left[I^{k} g\left(E^{*}\right)\right]
$$

Proof. Again it suffices to consider only Borel sets $B$ in $R^{n}$, and Borel subsets $B^{*} C B$. First, let $\theta$ be the family of sets $C$ such that $C$ is the union of a finite number of spheres of rational center and rational radius in $R^{n} . \theta$ is a countable family. If $F$ is a compact subset of $R^{n}$, then there exists $C_{1} \supset C_{2}$ $\supset \cdots, C_{i} \in \theta, F=\bigcap_{i=1}^{\infty} C_{i}$. We may further assume that $B$ is bounded. Let

$$
\theta_{B}=\{E \mid E=B \cap C, C \in \theta\} .
$$

$\theta_{B}$ is countable. For each $E$ in $\theta_{B}$, there is a $Z_{E}$ in $\mathrm{Z}$ such that, for $g$ in $G_{n}-Z_{E}$, 
$\Lambda(E) \geqq \Gamma\left[I^{k} g(E)\right]$. Set $Z=\cup Z_{E}$, for $E$ in $\theta_{B} . Z$ is in $Z$, and for $E$ in $\theta_{B}$ and $g$ in $G_{n}-Z$,

$$
\Lambda(E) \geqq \Gamma\left[I^{k} g(E)\right] .
$$

Now take $F$ closed with respect to $B$. Then there is a compact set $F^{*} \subset R^{n}$, such that $F=F^{*} \cap B$. Hence there is a sequence of sets $C_{1} \supset C_{2} \supset \ldots$ such that $F^{*}=\bigcap_{i=1}^{\infty} C_{i}$. Set $E_{i}=C_{i} \cap B . F=\bigcap_{i=1}^{\infty} E_{i}$, and $E_{1} \supset E_{2} \supset \cdots$. Also for all integers $i$, and for $g$ in $G_{n}-Z$,

$$
\Gamma\left[I^{k} g(F)\right] \leqq \Gamma\left[I^{k} g\left(E_{i}\right)\right] \leqq \Lambda\left(E_{i}\right) .
$$

Since $\lim _{i \rightarrow \infty} \Lambda\left(E_{i}\right)=\Lambda(F)$, we have, for all $g$ in $G_{n}-Z$,

$$
\Gamma\left[I^{k} g(F)\right] \leqq \Lambda(F) .
$$

Now let $O$ be open with respect to $B$. Then there is a bounded open set $O^{*}$ in $R^{n}$, such that $O=O^{*} \cap B$, and a sequence of compact sets $F_{1}^{*} \subset F_{2}^{*} \subset \cdots$, such that $O^{*}=\bigcup_{i=1}^{\infty} F_{i}^{*}$. Set $F_{i}=F_{i}^{*} \cap B . F_{i}$ is closed relative to $B$, and $O=\bigcup_{i=1}^{\infty} F_{i}$. For all integers $i$, and $g$ in $G_{n}-Z$,

$$
\Gamma\left[I^{k} g\left(F_{i}\right)\right] \leqq \Lambda\left(F_{i}\right) \leqq \Lambda(O) .
$$

Now $I^{k} g\left(F_{i}\right) \subset I^{k} g\left(F_{i+1}\right)$, and $I^{k} g\left(F_{i}\right)$ is $\Gamma$ measurable, and $I^{k} g(O)=\bigcup_{i=1}^{\infty} I^{k} g\left(F_{i}\right)$. So we have that $\Gamma\left[I^{k} g\left(F_{i}\right)\right] \rightarrow \Gamma\left[I^{k} g(O)\right]$ as $i \rightarrow+\infty$, and thus $\Gamma\left[I^{k} g(O)\right]$ $\leqq \Lambda(O)$. Now take $B^{*}$ a Borel subset of $B$. Fix $\epsilon>0$. Then there is a set $O$ open relative to $B$, such that $O \supset B^{*}$, and

$$
\Lambda(O)<\Lambda\left(B^{*}\right)+\epsilon \text {. }
$$

Then for $g$ in $G_{n}-Z$,

$$
\Gamma\left[I^{k} g\left(B^{*}\right)\right] \leqq \Gamma\left[I^{k} g(O)\right] \leqq \Lambda(O)<\Lambda\left(B^{*}\right)+\epsilon .
$$

Thus

$$
\Gamma\left[I^{k} g\left(B^{*}\right)\right] \leqq \Lambda\left(B^{*}\right)
$$

6.7. Lemma. For $E$ a subset of $R^{n}$, such that $\mu_{n}^{k}(E)<+\infty$, there is a set $Z$ in $\mathrm{Z}$ such that for $\mathrm{g}$ in $G_{n}-Z$,

$$
\stackrel{k}{\mu_{n}}(E) \geqq \int_{R^{k}} N\left(x^{\prime \prime}, I^{k} g, E\right) d L_{k} .
$$

Proof. Again it suffices to work with Borel sets $B \subset R^{n}$. By 6.6 there is a set $Z$ in $\mathrm{Z}$ such that for all Borel subsets $B^{*} \subset B$, and $g$ in $G_{n}-Z, \mu_{n}^{\boldsymbol{z}}\left(B^{*}\right)$ $\geqq L_{k} I^{k} g\left(B^{*}\right)$. For $i$ an integer, $R^{n}$ may be written as the union of a countable family of disjoint Borel sets $B_{i j}$, where $d\left(B_{i j}\right)<1 / i$. For $x^{\prime \prime}$ in $R^{k}$, let $f_{i j}\left(x^{\prime \prime}\right)$ be the characteristic function of $I^{k} g\left(B \cap B_{i j}\right)$, and let $f_{i}\left(x^{\prime \prime}\right)=\sum_{i=1}^{\infty} f_{i j}\left(x^{\prime \prime}\right)$. Then 


$$
\begin{aligned}
\int_{R^{k}} f_{i}\left(x^{\prime \prime}\right) d L_{k} & =\sum_{j=1}^{\infty} \int_{R^{k}} f_{i j}\left(x^{\prime \prime}\right) d L_{k}=\sum_{j=1}^{\infty} L_{k}\left[I^{k} g\left(B \cap B_{i j}\right)\right] \\
& \leqq \sum_{j=1}^{\infty} \mu_{n}^{k}\left(B \cap B_{i j}\right)=\mu_{n}^{k}(B) .
\end{aligned}
$$

Since $f_{i}\left(x^{\prime \prime}\right) \rightarrow N\left(x^{\prime \prime}, I^{k} g, B\right)$ as $i \rightarrow+\infty$, the result follows from the lemma of Fatou.

6.8. Lemma. Let $E$ be a k-rectifiable set in $R^{n}$. Then

$$
H^{k}(E)=\mu_{n}^{k}(E) \text {. }
$$

Proof. Again it suffices to work with Borel sets $B$. Let $Q$ denote the unit cube in $R^{k}$, that is, for $x$ in $R^{n}$, and $x$ in $Q$, and $x=\left(x_{1}, x_{2}, \cdots, x_{n}\right)$, we have

$$
0 \leqq x_{i} \leqq 1, \quad \text { for } 1 \leqq i \leqq k,
$$

and

$$
x_{i}=0, \quad \text { for } k+1 \leqq i \leqq n .
$$

Now $B$ may be taken as the image under a Lipschitz mapping of a subset of $Q$. Also it follows immediately from 6.5 that $\left(^{*}\right) H^{k}(B) \geqq \mu_{n}^{k}(B)$. By [6], this Lipschitz mapping may be extended to all of $Q$, with the same Lipschitz constant. Hence, in view of $\left({ }^{*}\right)$ without loss of generality, we may assume that

$$
B=T(Q), \quad T \text { a Lipschitz mapping. }
$$

Now let $u$ be any point of $Q$, and $g$ any element of $G_{n}$. Let

$J(u)$ be the Jacobian of $T$ at $u$;

$J_{0}^{*}(u)$ be the Jacobian of $g T$ at $u$;

$J_{o}(u)$ be the Jacobian of $I^{k} g T$ at $u$.

Now by ([7], 4.2), $J(u)=J_{0}^{*}(u)$. Also all of the above Jacobians exist $L_{k}$ almost everywhere on $Q$. Hence there is a Borel subset $E$ of $Q$ such that

(1) $T$ is univalent on $E$,

(2) All of the Jacobians exist and have positive, finite absolute values everywhere on $E$.

$$
\stackrel{k}{\mu_{n}}(B-T(E))=H^{k}(B-T(E))=0 .
$$

Now let $u$ be any point in $E$. By (2) there is a $k$-plane $\pi$ which is spanned by the $k$ column vectors of the differential matrix of $T$ at $u$. Select $g$ in $G_{n}$ such that $g(\pi)=R^{k}$. Then for this $g$,

$$
J(u)=J_{\theta}^{*}(u)=J_{\theta}(u) .
$$

Thus for $\epsilon>0$ there can be selecíed a sequence $g_{1}, g_{2}, \ldots$ in $G_{n}$ such that the sets 


$$
E_{i}^{*}=\left\{u|u \in E, 0 \leqq| J(u)-J_{0 i}(u) \mid<\epsilon\right\}
$$

cover $E$. Let

$$
E_{1}=E_{1}^{*}, \quad E_{i}=E_{i}^{*}-\bigcup_{j=1}^{i-1} E_{j}^{*} .
$$

Now, from 6.7 if necessary a second sequence $g_{1}^{\prime}, g_{2}^{\prime}, \ldots$ can be selected from $G_{n}$, such that for $u$ in $E_{i}$,

$$
0 \leqq\left|J(u)-J_{o i}(u)\right|<\epsilon,
$$

and

$$
\stackrel{k}{\mu_{n}}\left(T\left(E_{i}\right)\right) \geqq \int_{R^{k}} N\left(x^{\prime \prime}, I^{k} g_{i}^{\prime}, T\left(E_{i}\right)\right) d L_{k} .
$$

Hence, from ([7], 4.5 Theorem), we have

$$
\begin{aligned}
H^{k}(B) & =\int_{E} J(u) d L_{k}=\sum_{i=1}^{\infty} \int_{E_{i}} J(u) d L_{k} \leqq \sum_{i=1}^{\infty} \int_{E_{i}} J_{O_{i}^{\prime}}(u) d L_{k}+\epsilon \\
& =\sum_{i=1}^{\infty} \int_{R_{k}} N\left(x^{\prime \prime}, I^{k} g_{i}^{\prime}, T\left(E_{i}\right)\right) d L_{k}+\epsilon \leqq \sum_{i=1}^{\infty} \underset{\mu_{n}}{k}\left(T\left(E_{i}\right)\right)+\epsilon=\stackrel{k}{k}(B)+\epsilon .
\end{aligned}
$$

Hence

$$
H^{k}(B) \leqq \mu_{n}^{k}(B)
$$

\section{The decomposition theorem.}

7.1. Lemma. Let $A$ be a closed $\left(\mu_{n}^{k}, k\right)$ unrectifiable set in $R^{n}$, such that $\mu_{n}^{k}(A)<+\infty$. Then $\mu_{n}^{k}(A)=0$.

Proof. For any $g$ in $G_{n}$,

$$
\begin{gathered}
A=\left[A-\left(\bar{W}\left(\mu_{n}^{k}, A, g\right) \cup \bar{X}\left(\mu_{n}^{k}, A, g\right) \cup \bar{V}(A, g)\right)\right] \\
\cup\left[\bar{W}\left(\mu_{n}^{k}, A, g\right) \cup \bar{X}\left(\mu_{n}^{k}, A, g\right) \cup \bar{V}(A, g)\right] .
\end{gathered}
$$

By 3.15

$$
\stackrel{k}{\mu_{n}}\left[A-\left(\bar{W}\left(\mu_{n}^{k}, A, g\right) \cup \bar{X}\left(\mu_{n}^{k}, A, g\right) \cup \bar{V}(A, g)\right)\right]=0,
$$

for $\sigma_{n}$ almost every $g$ in $G_{n}$. By 6.4 and 6.6,

$$
L_{k}\left[I^{k} g\left(A-\left(\bar{W}\left(\mu_{n}^{k}, A, g\right) \cup \bar{X}\left({ }_{\mu_{n}}^{k}, A, g\right) \cup \bar{V}(A, g)\right)\right)\right]=0,
$$

for $\sigma_{n}$ almost every $g$ in $G_{n}$. Likewise by 4.2 and 6.6 and 6.4 , we have that 


$$
L_{k}\left[I^{k} g\left(X\left(\mu_{n}^{k}, A, g\right)\right)\right]=0,
$$

for $\sigma_{n}$ almost every $g$ in $G_{n}$. Likewise by 5.1 ,

$$
L_{k}\left[I^{k} g\left(\bar{W}\left(\mu_{n}^{k}, A, g\right)\right)\right]=0,
$$

for every $g$ in $G_{n}$. Likewise by 5.2 and 6.7 , we have that

$$
L_{k}\left[I^{k} g(\bar{V}(A, g))\right]=0,
$$

for $\sigma_{n}$ almost every $g$ in $G_{n}$. Hence, for $\sigma_{n}$ almost every $g$ in $G_{n}$,

$$
L_{k}\left[I^{k} g(A)\right]=0 \text {, }
$$

and therefore

$$
\stackrel{k}{\mu_{n}}(A)=0 \text {. }
$$

7.2. Theorem. I et $A$ be a Borel set in $R^{n}$ such that $\mu_{n}^{k}(A)<+\infty$. Then $A=A_{1} \cup A_{2}$, where $A_{1}, A_{2}$ are Borel sets. $A_{1} \cap A_{2}=\varnothing . A_{1}$ is countably k-rectifiable, and $\mu_{n}^{k}\left(A_{2}\right)=0$.

Proof. Let

$$
\gamma=\text { l.u.b. } \stackrel{k}{\mu_{n}}\left(A^{\prime}\right) \text {, }
$$

for $A^{\prime}$ a $k$-rectifiable subset of $A$. For each positive integer $i$, there is a $k$ rectifiable set $B_{i} \subset A$ such that

$$
\stackrel{k}{\mu_{n}^{k}}\left(B_{i}\right)>\gamma-1 / i
$$

Then there is a Lipschitz transformation from a bounded set $E_{i}$ in $R^{k}$ onto $B_{i}$. By [6] this transformation can be extended to the whole of $R^{k}$ with the same Lipschitz constant. If $F_{i}$ is a bounded closed set in $R^{k}$ containing $E_{i}$ then the intersection of $A$ and the image of $F_{i}$ under this extended transformation is a Borel $k$-rectifiable subset of $A$ containing $B_{i}$. Hence we may assume that $B_{i}$ is a Borel set for each $i$. Set

$$
A_{1}=\bigcup_{i=1}^{\infty} B_{i} .
$$

Then $A_{1}$ is a Borel and countably $k$-rectifiable set. The set $A_{2}=A-A_{1}$ is Borel and $\left(\mu_{n}^{k}, k\right)$ unrectifiable, $A=A_{1} \cup A_{2}, A_{1} \cap A_{2}=\varnothing$. Since $A_{2}$ is the union of a countable number of closed $\left(\mu_{n}^{k}, k\right)$ unrectifiable sets and a set of $\mu_{n}^{k}$ measure 0 , it follows from 7.1 that $\mu_{n}^{k}\left(A_{2}\right)=0$.

7.3. TheOREM. If $A$ is a Borel subset of $R^{n}$ and $\mu_{n}^{k}(A)<+\infty$, then $\mu_{n}^{k}(A)$ $=F_{n}^{k}(A)$, where $F_{n}^{k}$ is the integralgeometric Favard k-measure of $A$, in $R^{n}$. (See $[2], 2.18,5.11$ ) 
Proof. By 7.2, $A=A_{1} \cup A_{2}, A_{1} \cap A_{2}=\varnothing, A_{1}, A_{2}$ Borel, $A_{1}$ is countably $k$-rectifiable, and $\mu_{n}^{\mathbf{k}}\left(A_{2}\right)=0$. Thus

$$
F_{n}^{k}\left(A_{2}\right)=0 .
$$

Also by 6.8 and ([2], 5.14 Theorem),

$$
\stackrel{k}{\mu_{n}}\left(A_{1}\right)=H^{k}\left(A_{1}\right)=F_{n}^{k}\left(A_{1}\right) .
$$

7.4. Theorem. Let $m, k$ and $n$ be positive integers, $k<n<m$, and let $A$ be a Borel subset of $R^{n}$, which may be thought of as a subspace of $R^{m}$. Then

$$
\stackrel{k}{\mu_{n}}(A)=\stackrel{k}{\mu_{m}}(A) .
$$

Proof. Under the above assumptions Federer has shown $([8], 7)$ that

$$
F_{m}^{k}(A)=F_{n}^{k}(A) \text {. }
$$

To begin, we assume $\mu_{n}^{k}(A)<+\infty$. Then, by 6.8 and 7.2, $A=A_{1} \cup A_{2}, A_{i}$ Borel for $i=1,2, A_{1} \cap A_{2}=\varnothing$. Also $A_{1}$ is a countably $k$-rectifiable subset of both $R^{n}$ and $R^{m}$. So

$$
\stackrel{k}{\mu_{n}^{k}}\left(A_{1}\right)=H^{k}\left(A_{1}\right)=\stackrel{k}{\mu_{m}}\left(A_{1}\right), \text { and } \stackrel{k}{\mu_{n}}\left(A_{2}\right)=F_{n}^{k}\left(A_{2}\right)=0 \text {. }
$$

Hence $F_{m}^{k}\left(A_{2}\right)=0=\mu_{m}^{k}\left(A_{2}\right)$. Therefore $\mu_{n}^{k}(A)=\mu_{m}^{k}(A)$. We now wish to show that if $\mu_{n}^{k}(A)=+\infty$, then $\mu_{m}^{k}(A)=+\infty$. To show this it suffices to show that if $\mu_{m}^{k}(A)<+\infty$, then $\mu_{m}^{k}(A)=\mu_{n}^{k}(A)$. But this follows from exactly the same type of argument as used above.

\section{BIBLIOGRAPHY}

1. E. J. Mickle, On a decomposition theorem of Federer, Trans. Amer. Math. Soc. vol. 92 (1959) pp. 322-335.

2. H. Federer, The $(\phi, k)$ rectifiable subsets of $n$ space, Trans. Amer. Math. Soc. vol. 62 (1947) pp. 114-192.

3. E. J. Mickle and T. Rad6, Density theorems for outer measures in $n$ space, Proc. Amer. Math. Soc. vol. 9 (1958) pp. 433-439.

4. - On density theorems for outer measures, Interim technical report 3, The Ohio State University Research Foundation, 1958, p. 64.

5. A. P. Morse, $A$ theory of covering and differentiation, Trans. Amer. Math. Soc. vol. 55 (1944) pp. 205-235.

6. E. J. Mickle, On the extension of a transformation, Bull. Amer. Math. Soc. vol. 55 (1949) pp. $160-164$.

7. H. Federer, Surface area. II, Trans. Amer. Math. Soc. vol. 55 (1944) pp. 438-456.

8. _-, Dimension and measure, Trans. Amer. Math. Soc. vol. 62 (1947) pp. 536-547.

The Ohio State University,

Columbus, Ohio

UNIVERSITY OF KaNSAS

LAWRENCE, KaNSAS 